\title{
Diplomacia pública y América del Sur. De los conceptos a la práctica: Telesur y el caso venezolano
}

\author{
Érico SOUSA MATOS*
}

\begin{abstract}
Artículo recibido: 3 de marzo de 2015
Artículo aprobado: 16 de octubre de 2015

Doi: dx.doi.org/10.12804/desafios28.1.2016.10
\end{abstract}

Para citar este artículo: Sousa Matos, E. (2016). Diplomacia pública y América del Sur. De los conceptos a la práctica: Telesur y el caso venezolano. Desafíos, 28(I), 399-426. Doi: dx.doi. org/10.12804/desafios28.1.2016.10

\section{Resumen}

La Diplomacia Pública es el conjunto de métodos aplicados tanto por gobiernos, como por individuos y grupos privados en la conducción de las relaciones internacionales que buscan influir positivamente en la imagen y percepción de un Estado sobre el público general de otro país. En un contexto internacional en el cual el avance tecnológico y el proceso de globalización ha forzado la elaboración de nuevas estrategias de comunicación entre los Estados, este trabajo propone discutir los siguientes conceptos: Diplomacia Pública (Public Diplomacy), Diplomacia Mediática (Media Diplomacy), Diplomacia hecha por los medios (Media-Brokered Diplomacy) y Cyber-diplomacy aplicados al contexto de América del Sur a través del análisis del caso venezolano. Palabras clave: Poder, comunicación, diplomacia pública, medios de comunicación.

* Magíster Relaciones y Negociaciones Internacionales, Facultad Latinoamericana de Ciencias Sociales (FLASCO), sede Argentina. Correo electrónico: erico-matos@hotmail. com 


\title{
Public Diplomacy from Concept to Practice: Telesur and the Venezuelan Case
}

\begin{abstract}
Public diplomacy is defined as the conduct of international relations by governments, private individuals and groups through public media communications, trying to positively influence the perception of the country's image abroad. The technological progress and globalization have heralded the improvement of communication strategies among states. This paper discusses the following concepts: public diplomacy; media diplomacy; media-brokered diplomacy; and cyber-diplomacy as applied to the context of South America through the analysis of the case of Venezuela.
\end{abstract}

Keywords: Power, communication, public diplomacy, mass communication.

\section{Diplomacia pública e a América do Sul. Dos conceitos à prática: Telesur o caso venezuelano}

\begin{abstract}
Resumo
A Diplomacia Pública é o conjunto de métodos aplicados tanto por governos, quanto por individuos e grupos privados na condução das relações internacionais que buscam influir positivamente na imagem e percepção de um Estado sobre o público geral de outro pais. Em um contexto internacional no qual, o avance tecnológico e o processo de globalização tem forçado a elaboração de novas estratégias de comunicação entre os Estados, este trabalho propõe discutir os seguintes conceitos: Diplomacia Pública (Public Diplomacy), Diplomacia Mediática (Media Diplomacy) e Diplomacia feita pelos meios (Media-Broker Diplomacy) e Cyber-diplomacy aplicados ao contexto da América do Sul através da análise do caso venezuelano; estados que têm aplicado o uso de tais métodos na maneira de condugir as suas relações internacionais.
\end{abstract}

Palavras -chave: Poder, comunicação, diplomacia pública, meios de comunicação 


\section{Introducción}

La diplomacia es comprendida como el arte de negociar entre los Estados utilizando prácticas y métodos que buscan de manera directa comunicarse y/o ejercer influencia entre uno y otro, sin recurrir a la fuerza.

Así que con fines didácticos en este artículo utilizaré la división del concepto "diplomacia" propuesta por Nancy Snow en su texto "Rethinking Public Diplomacy", y dividiré ese concepto en dos. El primero será aquel que denominamos diplomacia tradicional, que es el método donde prevalece las relaciones gobierno-gobierno, esto es, a lo que se denomina modelo G2G (Snow, 2008, p. 6), donde no existe contacto directo con los ciudadanos del otro Estado. Todas las relaciones ocurren exclusivamente a nivel gubernamental, de manera técnica y, en muchos casos, cerradas. Sin embargo, a su vez Nancy Snow denomina que existe otra vertiente que ha surgido a lo largo del siglo XX, la cual es denominada diplomacia pública, que en su definición clásica es: “(...) public diplomacy has been about governments talking to global publics (G2P), and includes those efforts to inform, influence, and engage those publics in support of national objectives and foreign policies”. (Snow, 2008, p. 6). Así, en este modelo G2P, las relaciones diplomáticas entre los Estados no son más conducidas meramente entre gobierno-gobierno; la opinión pública y los ciudadanos son actores que pasan a ser relevantes en esa relación.

Partiendo de lo señalado por Nancy Snow, en este artículo desarrollaré el modelo G2P de diplomacia pública al que la autora hace referencia. Para eso, voy enfocarme en los medios de comunicación internacionales y analizaré el caso de venezolano.

La tendencia a la democratización de las sociedades y el creciente interés por la política internacional ha transformado el modelo de diplomacia tradicional, esto se debe a la presencia de diversos actores sociales, como las $\mathrm{ONG}$, las cuales son grupos privados que, de alguna manera, buscan influir y participar en las decisiones tomadas sobre política exterior (Martínez Pandiani, 2006, p. 15). Por lo tanto, 
los temas internacionales son discutidos en la cotidianidad y han transformado las discusiones sobre política exterior en algo más cercano al ciudadano. Inclusive, el debate sobre esos temas influye fuertemente en el escenario político local de diversos países.

De esta manera, los Estados han adoptado medios de promover una presencia y una imagen internacional favorable, utilizando los medios de comunicación y radiodifusión, así como llevando a cabo la promoción de eventos culturales y deportivos, además de suscitar la organización de intercambios académicos con el objetivo central de promover la construcción de una relación entre los Estados y los ciudadanos de otros países, más allá de las relaciones gubernamentales (Nye, 2008, p. 94). Este conjunto de acciones es lo que algunos autores llaman "diplomacia pública".

El desarrollo de nuevas tecnologías de la información y comunicación ha acortado las distancias entre los distintos pueblos y Estados. A su vez, Delaney (1968) señala que la "Diplomacia Tradicional" ha sufrido enormemente al sumarse a las modernas redes de comunicación masiva, que propiciaron una comunicación veloz entre las más diversas regiones del mundo posibilitando el traspaso fácil de las fronteras de los Estados nacionales.

Estos procesos ocasionaron el surgimiento de nuevas dinámicas frente a la diplomacia tradicional que todavía persiste como principal modelo, especialmente en los países en vías de desarrollo. Aún existe cierto escepticismo en las cancillerías de nuestra región sobre la importancia de desarrollar la diplomacia pública a través del modelo G2P como herramienta de promoción de política exterior. Sin embargo, algunos Estados en América del Sur han buscado diversificar su influencia internacional en los últimos años utilizando diferentes medios y modelos de diplomacia. El uso de los medios tradicionales de comunicación gobierno-gobierno (G2G), como conferencias y reuniones de cancilleres, fue ampliado en la región, así como el uso de internet (cyber-diplomacy) y de la televisión (diplomacia mediática) con el objetivo de tener nuevas maneras de comunicarse con el público extranjero, para informar, influir e involucrar a esos públicos 
en apoyo de sus objetivos en los temas de política internacional. Modelo este que argumenta Nancy Snow como clásico en la diplomacia pública (G2P).

Por lo tanto, invariablemente, la diplomacia pública está vinculada al poder de un país y, particularmente, al "softpower" de un Estado, según señala Nye (Nye, 2008); sin embargo, no es exclusivamente el Estado el que es capaz de conducir acciones en cuanto a la diplomacia pública; la participación de la sociedad civil y las organizaciones privadas tiene un papel importante en la búsqueda de apoyo en los públicos extranjeros sobre la política internacional de un Estado.

Sin embargo, Snow argumenta que, por ser un elemento que compone el llamado "softpower" de un país, "thus, the term "soft" can be misleading to some scholars and practitioners of public diplomacy who view what we do in almost messianic terms" (2009, p. 3). Por lo tanto, es necesario entender la definición de Nye del concepto "poder" la cual es: "(...) is the ability to influence the behavior of others to get the outcomes one wants. But there are several ways to affect the behavior of others. You can coerce them with threats; you can induce them with payments; or you can attract and co-opt them to want what you want." (2004, p. 2). De esta manera, se puede afirmar que, conceptualmente, "softpower" está basado en tres recursos de poder de un Estado; el primero es su cultura (en los lugares en que esto es atractivo para los demás), seguido de sus valores políticos (esto tanto en política nacional, como en el extranjero), y en tercer lugar, su política exterior (cuando se ve como legítimo y tiene autoridad moral) (Nye, 2008). Luego, el termino "soft' en softpower no es, según argumentan los autores, un termino "blando"; por lo contrario, puede representar una política agresiva. Esto es lo que el profesor Raymond Aron llama "the war of propaganda" en su libro Guerra y Paz (2003).

Los medios de comunicación constituyen una fuente de recursos simbólicos muy importantes en el análisis que propongo en este texto. Es a través de los medios de comunicación que las sociedades modernas interactúan en un proceso continuo de intercambio de información. Así, en este escenario los recursos simbólicos y la forma como imaginamos el mundo son elementos constitutivos de 
poder internacional, debido a que, como señala Walker "sugerir que la cultura y la ideología son cruciales para el análisis de la política mundial no es necesariamente adoptar posiciones idealistas (...) Al contrario es importante reconocer que las ideas, la conciencia, la cultura y la ideología están ligadas al tipo más inmediatamente visibles del poderpolitico, military económico" (citado por Porcelli, 2013, p. 71). Por lo tanto, los medios de comunicación son el principal instrumento por el cual se transmiten los recursos simbólicos y culturales de un Estado hacia otro.

La transmisión en tiempo real de los acontecimientos globales, a través de las nuevas tecnologías de la información y comunicación (TCI), ha impactado enormemente el proceso de toma de decisiones de los Estados en relación con la política exterior, ya que, la televisión e internet auxilian a los diplomáticos en el momento de emitir mensajes hacia el extranjero, los cuales llegan a su público de forma prácticamente instantánea (Martínez Pandiani, 2006), además de servir como instrumento de difusión del recurso simbólico y el "softpower" de un Estado, aunque esto puede, paradójicamente, ocasionar efectos indeseables, conforme afirmó el exsecretario de Estado James A. Baker, sobre la presencia de los medios de comunicación en la política internacional "is to drive policymakers to have a policy position. I would have to articulate it very quickly. You are in a real-time mode. You don't bave time to reflect' (citado por Livingston, 1997, p. 2).

Sin embargo, según señala Eytan Gilboa (2000), en los últimos años el público no acepta tomar una actitud pasiva ante las decisiones internacionales, sin tener un mínimo de información sobre los temas negociados entre las partes; en este contexto, los medios de comunicación sirven para suministrar el tipo de información demandada de manera muy sencilla. Esto representa un cambio y nuevos desafíos en la estructura gubernamental, especialmente, en los países en desarrollo.

Este artículo está divido en dos partes. Inicialmente son abordados los conceptos teóricos de la diplomacia pública y, especialmente, cómo han sido utilizadas las redes de televisión internacional para informar, influir e involucrar a la opinión pública internacional. Parto de la interpretación de que los Estados modernos invierten en 
comunicación internacional directa con el objetivo de lograr apoyo a sus demandas y obtener capital político internacional (G2P). En la segunda parte se busca retratar el uso de estos modelos a un análisis del contexto regional a través del estudio del caso venezolano y la Nueva Televisión del Sur (TELESUR).

\section{La nueva diplomacia}

En la diplomacia moderna es tan importante hacer y conducir las acciones diplomáticas, como emitir mensajes a escala global donde estén reflejadas dichas acciones promovidas por los gobiernos en temas internacionales (Nieto \& Peña, 2008). De manera que aquellos actores que hasta entonces estaban alocados por debajo del Estado Nacional, sin participación en temas relevantes del escenario internacional, como es el caso de los medios de comunicación, pasan a tener una postura de mayor relevancia.

La diplomacia pública, por lo tanto, es el conjunto de métodos aplicados tanto por los gobiernos, como por individuos y grupos privados que buscan influir positivamente en la imagen y percepción de un Estado de manera directa sobre el público general de otro país.

Según señala Nye (2008), es en el inicio del siglo XX a partir de 1920 que surge esto, favorecido a través del fuerte desarrollo de la radio como principal medio de comunicación internacional. Durante estos años, la intensa máquina de propaganda que había sido desarrollada durante la Primera Guerra Mundial pasa a la radio, lo que ocasiona que diversos gobiernos busquen ingresar en el ámbito de la radiodifusión internacional y promover la defensa de sus intereses frente al público global. Así, en la década de 1930 se hace evidente la disputa existente entre los gobiernos socialistas y los fascistas para promover imágenes favorables de sus regímenes a los públicos extranjeros (Nye, 2008, p. 97); esto lo lograban utilizando el cine, la radio y la imagen como principal instrumento. Por lo tanto, es en ese periodo que los Estados pasan a mirar la capacidad de influir la opinión pública internacional como una herramienta de poder. 
Es durante inicio del siglo pasado cuando surge en los países de habla inglesa el creciente interés por los temas de política internacional. Según señala Carr (2001), este público inicialmente cuestionaba los tratados secretos acusándolos como los principales responsables de la primera gran guerra. El movimiento en contra de estos acuerdos secretos es de enorme importancia, ya que demostraba el creciente interés público por los temas de política internacional y anunciaba el nacimiento de una nueva ciencia social, las relaciones internacionales (Carr, 2001). Los ciudadanos y la opinión pública empiezan a querer participar, de algún modo, en los negocios internacionales.

Actualmente no es posible asumir que las cancillerías son los únicos organismos en el Estado que conservan el monopolio exclusivo de las relaciones exteriores, (Martínez Pandiani, 2006, p. 27) ni los únicos detentores de la elaboración o contacto en temas relacionados a política externa. ${ }^{1}$

Eytan Gilboa en el artículo titulado "Diplomacy in the media age: Threemodels of uses and effects" (2001) propone conceptualmente una manera de abordar el estudio de este tema, donde divide en tres segmentos el modo en que un país direcciona su discurso hacia un público extranjero: la diplomacia pública (public diplomacy), la diplomacia mediática (media diplomacy) y la Diplomacia hecha por los medios (media-broker diplomacy). En este estudio agregué el nuevo concepto de cyber-diplomacy, para que sea posible tener una comprensión más amplia sobre la realidad en América del Sur.

\subsection{Diplomacia Pública}

El término diplomacia pública fue utilizado por primera vez en 1965 con el establecimiento del Centro de Diplomacia Pública Edward R. Murrow, de la Universidad Tufts, en Boston. (Nieto \& Peña, 2008). El Diccionario de Relaciones Internacionales promovido por el Departamento de Estado estadounidense señala que: "Diplomacia

1 Ver Frisancho, J.R.C. (2010). 
pública se refiere a los programas patrocinados por el gobierno destinados a informar e influir en la opinión pública de otros países. Sus herramientas principales son publicaciones (diarios, revistas y libros), películas, intercambios culturales, la radio y la televisión" (U.S Depto. State, citado por Nieto \& Peña, 2008, p. 155).

Mientras que la diplomacia tradicional se enfoca en la formalización de las relaciones entre los Estados, la diplomacia pública busca exceder las comunicaciones exclusivamente gubernamentales. Por lo tanto, es posible entender el carácter de propaganda de la diplomacia pública, puesto que, utilizando diversos medios, el Estado o las personas privadas (grupos de influencia) buscan influir de manera directa o indirecta en las actitudes y toma de decisiones en política exterior de otros Estados. (Signitizer, citado por Gilboa, 2001, p. 8).

\subsection{Diplomacia Mediática}

En la diplomacia mediática, los medios de comunicación tienen una importancia más relevante. En la diplomacia pública se utiliza a los medios como agentes de difusión del contenido elaborado, mientras que en la diplomacia mediática los medios no son simples transmisores de información, son instrumentos de negociación con objetivos claros, ya que pueden establecer una conexión entre los Estados y actores no Estatales que puede auxiliar en el avance de las negociaciones (Martínez Pandiani, 2006, p. 73). Gilboa clasifica el uso de diplomacia mediática como opción para un conflicto ya existente, como una herramienta que puede auxiliar a negociar o solucionar el problema (Gilboa, 2001).

De modo que los medios desarrollan un papel fundamental en la comunicación entre los Estados, ejemplo de esto es el caso de los rehenes en la embajada estadounidense secuestrados en Teherán, donde se evidenció una amplia utilización de los medios de comunicación entre Estados Unidos y los secuestradores para dialogar, o cuando en 1990 el secretario de estado estadounidense, James Baker, envía un ultimátum a Saddam Hussein en lugar de enviarlo a través de la Embajada de Estados Unidos en Bagdad (Burity, 2012, p. 10). 


\subsection{Diplomacia hecha por los medios de comunicación}

El tercer y más polémico modelo que Gilboa propone es aquel en el que los medios de comunicación actúan como un verdadero actor en las relaciones internacionales. Este modelo considera a los medios de comunicación y a los periodistas como el cuarto "poder", que puede trabajar en las negociaciones internacionales. Hasta entonces los conceptos anteriores consideran aún que es el Estado el principal conductor de la política exterior, en el cual, los periodistas están ubicados en su función tradicional, es decir relatar a los hechos internacionales, más no influir en la política exterior.

Gilboa (2005) pasa a considerar a los medios de comunicación como un actor que no solamente relata, sino que actúa en la política exterior de los Estados, y argumenta que eso ocurriría típicamente "when there is no contact between enemies and no third party to help them resolve their differences. Larson (1988) observed that television provides an interactive channel for diplomacy which is instantaneous or timely and in which journalists frequently assume an equal role with officials in the diplomatic dialogue" (p. 99).

\subsection{Cyber-diplomacy}

Es notable el incremento de las comunicaciones internacionales a través de internet, lo cual acorta las distancias físicas y facilita la interacción entre diversos actores, especialmente, después del ascenso de las redes sociales en los últimos años. Así el académico Pierre C. Pahlavi describe el surgimiento de la Cyber-diplomacy, una batalla de persuasión que se intensificó en la Era Digital a través de una "guerra electrónica", donde los Estados han utilizado modernas técnicas de persuasión con el bombardeo de imágenes y videos para construir una esfera que pueda influenciar las mentes de las poblaciones extranjeras (Pahlavi, 2003).

Por lo tanto, esto representa nuevos desafíos para las cancillerías de los países en vías de desarrollo que debido a cuestiones estructurales provocadas por limitación presupuestaria no logran alcanzar suplir 
al crecimiento continuo de demanda sobre temas internacionales lo que ocasiona cierta vulnerabilidad frente a posibles amenazas proveniente del exterior.

\subsection{La imagen y el poder internacional}

Según señala Martínez Pandiani (2006) citando a Walter Lippmann; "la imagen transmitida a través del televisor, representa la forma más segura de transmitir una idea" (Martínez Pandiani, 2006, p. 96). Así, cuando el televisor pasa a tener más espacio como principal medio de comunicación frente a la radio y los periódicos impresos. Ocurre el proceso denominado por algunos estudiosos "mediatización" de las relaciones internacionales. Este fenómeno es señalado por algunos autores, como Pandiani y Eliseo Verón, como la irrupción de la televisión a través de la fuerza de la imagen transmitida que ocasiona profundos cambios en la sociedad y en la manera de gestionar sus conflictos sociales y políticos. Esto significa que en cuanto a las relaciones internacionales, representa un nuevo paradigma a la hora de comunicar y negociar debido a la presencia de los medios masivos de comunicación son la fuente más consultada por los ciudadanos y agentes políticos en la hora de toma de decisiones políticas (Martínez Pandiani, 2006, pp. 94-95).

El profesor Raymond Aron, en su libro Pazy Guerra (2003) habla sobre la guerra de la propaganda, que es un nuevo elemento en nuestro siglo. Por lo tanto, los Estados buscan nuevos métodos para ampliar, mantener o ganar poder a nivel internacional, sin utilizar de manera exclusiva el uso exclusivo de elementos que componen el llamado "hardpower" para lograr esos objetivos.

A new factor, in our century -one implied by our democratic customs- is that the masses are courted just as much as the leading minorities by the words and the spokesmen of the aggressive states. Each of the blocs, each of the giants, attempts to convince the governed, on the other side of the line of demarcation, that they are exploited, oppressed, abused. The war of propaganda, the war of radio, marks the permanence of the conflict among 
states and the ceaseless recourse to means of pressure. In this interplay, power is not a function of military strength or economic resources. One regime is better suited for export through advertising, another state is better able to recruit disinterested representatives or more willing to supply money for the rape of conscience. (Aron, 2003, p. 60)

Mientras tanto no es posible hablar de que existan elementos que permitan identificar la existencia de una war of propaganda y que las redes de televisión internacional sirvan únicamente para este hecho. Sin embargo, en los últimos años el mundo presenció el surgimiento de diversos canales internacionales de televisión que son de manera directa o indirecta patrocinados por diversos Estados nacionales (Painter, 2006, p. 5) como Al-jazeera international, de Qatar, Russia Today-(RT), de Rusia, y France 24, de Francia, todas fundadas en 2005. También destacan Telesur, promovida por Venezuela con el apoyo de los Estados miembros de la Alianza Bolivariana para los Pueblos de Nuestra América (ALBA) además de otros gobiernos de América del Sur como Argentina y Uruguay también en 2005, y Euronews, cadena que fue fundada por los Estados miembros de la Unión europea (UE) en 1992.

Según el levantamiento adaptado a partir de la investigación de Rai y Cottle (2007), señalo las existencia de aproximadamente 125 canales internacionales de noticias, los cuales en 2014 tienen su señal transmitiendo desde un país hacia otros; mientras tanto, lo más sorprendente, es la inmensa cantidad de canales que tienen presencia de los Estados nacionales en su capital o que son directamente propiedades de los Estados nacionales. En el inicio de los años noventa existían 2 canales con estas características que denomino de "canales públicos internacionales". En 2014 este número era de cuarenta y cinco canales entre los cuales están cuarenta y uno que transmiten en el formato " $24 / 7$ de noticias" y cuatro canales que transmiten información y entretenimiento, haciendo uso de un formato conocido como infotrainment. 
Gráfico 1. Número de canales de noticias internacionales

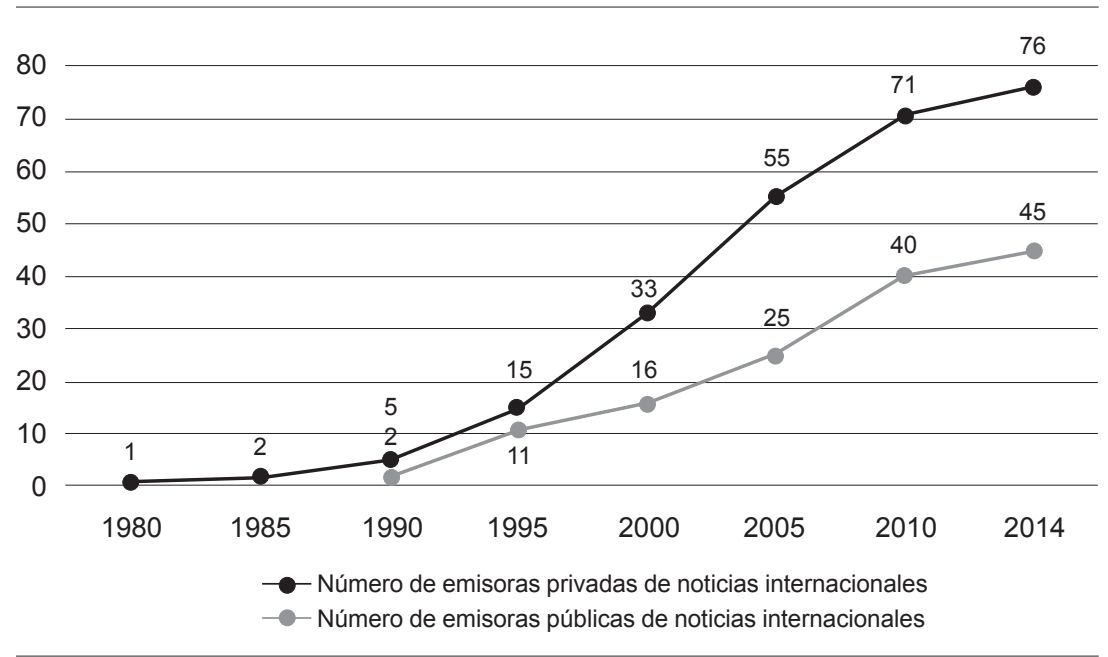

Fuente: elaboración propia, adaptado a partir de Rai y Cottle (2007).

El escenario internacional de la comunicación es dominado por corporaciones mediáticas privadas; sin embargo, entre los años 2005 y 2010 se nota un aumento exponencial en la participación de los Estados en los medios de comunicación internacionales; mientras los medios privados registran un aumento de $29 \%$ en este periodo en la apertura de nuevas empresas, el número de emisoras públicas registra un crecimiento de $60 \%$. Aunque las corporaciones privadas mediáticas representan en números absolutos un dominio de este escenario, según señala Nye, sin la existencia de relación directa con el gobierno, estas corporaciones son representantes naturales de sus Estados y funcionan como instrumento difusores del "softpower" de sus respectivos Estados (Nye \& Owens, 1996 p. 29). Por más que estas empresas aparentan una característica transnacional, las cadenas internacionales generalmente no poseen grandes estructuras e inversiones fuera del país en que mantienen su sede central, además que no es común que los cuadros profesionales como técnicos, directivos y periodistas sean de otra nacionalidad (Martínez Pandiani, 2006, p. 97).

Así, promover una política de comunicación internacional eficaz es especialmente complejo para los países en desarrollo, inclusive, entre los Estados de poder mediano. Pocos Estados en el mundo han con- 
seguido elaborar una estructura estatal institucional como la de los Estados Unidos, que ejerce a través de la Subsecretaría de diplomacia pública formada debido a la preocupación de los Estados Unidos respecto a su imagen internacional y que se dedica, conforme informaciones publicadas en la página web del Departamento de Estado a: "promover los intereses nacionales, y mejorar la seguridad nacional, al informar e influenciar la opinión pública extranjera a través de la ampliación y el fortalecimiento de la relación entre el pueblo y el Gobierno de los Estados Unidos y los ciudadanos del resto del mundo"2 (Under Secretary for Public Diplomacy and Public Affairs, [en línea]).

América del Sur no ha quedado afuera de este nuevo fenómeno que ha emergido en las relaciones internacionales. Así, aunque enfrentando limitaciones presupuestarias, los Estados han desarrollado nuevas maneras de hacer política exterior con diferentes enfoques y estrategias. Uno de los principales motivos de este interés en desarrollar una política exterior proactiva es debido al peso cada vez mayor que las variables externas tienen sobre las políticas nacionales. Por lo tanto, es necesario para el Estado lograr establecer una Diplomacia Pública eficaz. Sin embargo, no es una tarea fácil; como señalan los autores Hachten y Scotton se ha observado en la historia que usualmente los Estados en desarrollo no producen con éxito políticas de diplomacia pública ni de comunicación internacional, pero sí las reciben de los Estados desarrollados (Hachten \& Scotten, 2002, p. 14).

\footnotetext{
2 The mission of American public diplomacy is to support the achievement of U.S. foreign policy goals and objectives, advance national interests, and enhance national security by informing and influencing foreign publics and by expanding and strengthening the relationship between the people and Government of the United States and citizens of the rest of the world.

The Under Secretary for Public Diplomacy and Public Affairs Richard Stengel leads America's public diplomacy outreach, which includes communications with international audiences, cultural programming, academic grants, educational exchanges, international visitor programs, and U.S. Government efforts to confront ideological support for terrorism. The Under Secretary oversees the bureaus of Educational and Cultural Affairs, Public Affairs, and International Information Programs, as well as the Center for Strategic Counterterrorism Communications, and participates in foreign policy development.
} 
No obstante, en América del Sur, el gobierno de Hugo Chávez ha implementado nuevas maneras de conducir la política internacional en su país, pero, el modelo de diplomacia tradicional no ha perdido espacio en la política exterior de Venezuela que aún conducen sus relaciones fuertemente basada sobre este modelo tradicional de diplomacia. Pero, cabe destacar que es en ese país el uso de nuevas estrategias de comunicación internacional y diplomacia ha sido más destacado en comparación con los demás países de la región.

\section{Telesur y el caso venezolano}

En este artículo propongo un análisis del caso venezolano y cómo ese país ha utilizado una gama amplia de instrumentos que buscan aumentar la presencia y la imagen favorable a Venezuela en el exterior, desde la elección de Hugo Chávez en 1999.

Chávez asume el gobierno venezolano el 2 de Febrero de 1999. Ocho años antes, el entonces teniente coronel Hugo Chávez llevó a cabo un intento infructuoso de golpe de Estado, del que él fue el líder principal. Pero, el discurso de pocos segundos de Chávez en vivo a través de la televisión nacional a todo el país donde anuncia el derrocamiento del Movimiento Militar Bolivariano y sus aliados, proyectará a Chávez nacionalmente (Cañizález \& Lugo, 2007, 53).

Compañeros, lamentablemente, por ahora, los objetivos que nos planteamos no fueron logrados en la ciudad capital. Es decir, nosotros acá en Caracas, no logramos controlar el poder. Ustedes lo hicieron muy bien por allá, pero ya es tiempo de evitar más derramamiento de sangre. Ya es tiempo de reflexionar y vendrán nuevas situaciones y el país tiene que enrumbarse definitivamente hacia un destino mejor. Así que oigan mi palabra. Oigan al Comandante Chávez quien les lanza este mensaje para que, por favor, reflexionen y depongan las armas porque ya, en verdad, los objetivos que nos hemos trazado a nivel nacional, es imposible que los logremos. Compañeros, oigan este mensaje solidario. Les agradezco su lealtad, les agradezco su valentía, su desprendimiento, y yo, ante el país y ante ustedes, asumo la responsabilidad de 
este Movimiento militar Bolivariano. Muchas gracias (Chávez, alocución del 4 de Febrero de 1992).

En un país donde la población siempre estuvo acostumbrada a que la clase política se eximía de culpa, la acción de Chávez generó ante gran parte de los venezolanos, cierta popularidad y respeto (Valente \& Santoro, 2006, p. 7). Chávez era desconocido para el público y pasó a ser un rostro conocido, debido a eses pocos segundos en que tuvo presencia en la televisión venezolana en el día 4 de Febrero de 1992. Por lo tanto, Chávez sabía sobre la importancia de los medios de comunicación para todo proceso de reformas que conducirá a partir de su elección en 1999.

En abril de 2002 Chávez sufre un intento de Golpe de Estado. Según señalan los profesores brasileños Leandro Valente \& Mauricio Santoro (2006), la actuación lenta de los medios internacionales para clasificar lo ocurrido en Venezuela como un golpe de Estado, buscaba construir una imagen de tranquilidad del país, por eso, en un primero momento el Golpe de Estado era anunciado a través los medios de comunicación internacional, pero no como tal, sino que informaban que el presidente Chávez había dejado el poder por su voluntad (Valente \& Santoro, 2006, p. 9).

Así que es posible considerar que la visión de mundo del Militar Hugo Chávez, profundamente sesgada a una visión Geopolítica (Serbin, 2010, p. 18), hace que después de los ocurridos en Abril de 2002 sea necesario la construcción en el exterior de una imagen positiva del país a través de un medio directo de información que posibilite a Venezuela hablar al mundo sin la presencia de intermediarios.

\subsection{Venezuela y la diplomacia pública}

Por lo tanto, como se ha dicho, la opinión pública internacional pasó a ser disputada por los Estados en busca de respaldo, capital político y apoyo a sus demandas. Mientras, esa práctica es corriente en los países desarrollados, es relativamente reciente que gobiernos en nuestra 
región busquen establecer un medio internacional de comunicación que colabore en la proyección del país.

Además de eso, en 2005 el gobierno venezolano optó por utilizar la diplomacia pública como medio de promover publicidad internacional. Este fue el caso del patrocino a través de la PDVSA a la escuela de samba "Unidos de Vila Isabel", que se consagró campeona del carnaval de Rio de Janeiro en este año. El tema elegido fue "Soy loco por ti, America", haciendo referencia a las maravillas naturales y culturales de América Latina y la importancia de la integración de los pueblos de nuestra región (Valente \& Santoro, 2006, p. 11). Según Valente \& Santoro (2006), PDVSA y el gobierno venezolano al patrocinar la escuela de Samba Vila Isabel no tienen el objetivo principal de vencer, pero sí el de llegar a tener la atención de los millones de televidentes de este evento, no solo en Brasil, sino en el mundo, para construir una imagen favorable del país (Valente \& Santoro, 2006, p. 12). Por lo tanto, buscando una imagen favorable del país.

En Venezuela la antigua Agencia de Noticia Venezolana "Venpres" fundada en el primer gobierno de Carlos Andrés Pérez (1974-1979) buscó crear e internacionalizar una agencia de noticias local con el "objetivo de darle a Venezuela la posibilidad de proyectarse geopolíticamente en el escenario mundial y ejercer diplomacia pública" (Cañizález \& Lugo, 2007, p. 59).

No obstante, bajo el gobierno de Hugo Chávez este proyecto resurge con objetivos similares, ahora, a través de la inauguración de La Nueva Televisión del Sur (TELESUR) en 2005 , empresa de televisión multiestatal fundada mediante un acuerdo inicial entre los Gobiernos de Venezuela, Argentina, Cuba y Uruguay. Surge bajo el argumento de romper con el monopolio de información ("guerra mediática") de las agencias de noticias estadounidenses y europeas sobre la región, posibilitando un canal de intercambio mayor de noticias referentes a los países de la región.

El control accionario de TELESUR es Venezuela con 46\%; le sigue Argentina con un $20 \%$, Cuba con un $19 \%$, Uruguay con un $10 \%$ y 
Bolivia con un 5\% de participación (Minci, 2006 citado por Cañizález \& Lugo, 2007, p. 57). Además de las recientes incorporaciones minoritarias de Ecuador y Nicaragua, países como Uruguay y Argentina aún tienen que cumplir con muchos de sus compromisos financieros (Cañizález \& Lugo, 2007, p. 57). El presupuesto destinado a los primeros años de operación del canal fue de 10 millones pagado integralmente a través de la Corporación Venezolana de Petróleo, empresa estatal filial de PDVSA (Últimas Noticias, citado por Cañizález y Lugo, 2007, p. 56).

\subsection{Venezuela y la Diplomacia mediática}

El gráfico 2 hace un análisis de los países nombrados en las noticias publicadas en los programas de "Edición Central" transmitidos durante la semana de 02 de febrero hasta 06 de febrero de 2015. El programa transmite desde Caracas a las 19:30 horas (GMT -4:30 Horas), con una duración promedio de una hora y veinte minutos, y es conducido por los periodistas Abraham Istillarte y Tatiane Pérez. Este programa ofrece el resumen de las noticias más importantes del día, además presenta entrevistas con expertos, quienes opinan sobre el tema más relevante del día y resalta la participación de los corresponsales internacionales del canal que transmiten una visión sobre el tema.

\section{Gráfico 2. Países nombrados en las noticias publicadas}

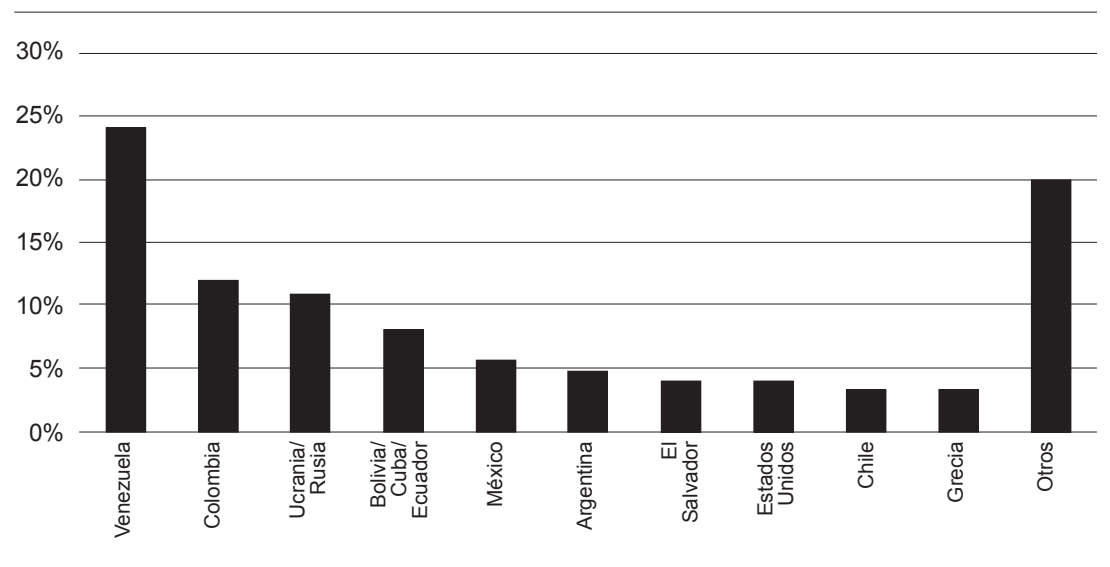

Fuente: elaboración propia. 
Según el gráfico, Venezuela es el país que más registra presencia en las noticias publicadas en el programa analizado con $24 \%$, de las noticias, seguido por Colombia con 12\% y las noticias del conflicto en el este europeo con $11 \%$. Sobre el canal pesan características que podemos relacionar con los rasgos geopolíticos del expresidente Hugo Chávez; la cobertura de Telesur se concentra en dos ejes, geopolítico e ideológico, donde las noticias publicadas refuerzan esta idea: Colombia, debido a la proximidad geográfica tiene una participación relevante en las noticias publicadas, mientras que países como Bolivia, Ecuador y Cuba, aliados ideológicos tradicionales del gobierno venezolano, representan 8\% de las noticias emitidas en este programa. En el caso de Rusia, que debido al reciente conflicto en el Este europeo y la caída del precio del petróleo y del gas, se puede afirmar que la aparición de noticias sobre este país en este programa se debe en gran medida al acercamiento deposiciones ideológicas entre los gobiernos de Venezuela y Rusia en contra de Estados Unidos y Europa.

El gobierno venezolano ha demostrado a lo largo de los años que Telesur está en operación, y que está dispuesto a utilizar los medios de comunicación para promover una diplomacia pública a favor de la seguridad del País. Por lo tanto, diferente al proyecto de Venpress del expresidente de Carlos Andrés Pérez, el Chavismo, utiliza a Telesur como una herramienta de defensa de Venezuela, contrarrestando la influencia y la hegemonía internacional de los medios de comunicación

El gráfico 3 señala las noticias publicadas en las ediciones analizadas del programa Edición Central. En todas las ediciones algunos temas son recurrentes como la guerra económica; el conflicto en Ucrania y el proceso de paz en Colombia, los cuales tuvieron una representación de 39\% de las noticias publicadas en el periodo analizado, estando presentes en todas las ediciones. Estos tres temas señalan claramente la posición de propaganda ideológica y geopolítica del canal, especialmente, las noticias publicadas sobre la guerra económica en Venezuela, donde es recurrente el uso de expresiones como "no hay desabastecimiento" en el país, pero sí argumentan que existe un acaparamiento de productos promovido por sectores de oposición al gobierno nacional. Por lo tanto, claramente, Telesur busca transmitir 
internacionalmente la posición del gobierno venezolano en este tema, además, de incluir declaraciones de algunos jefes de Estados aliados a Venezuela, como Evo Morales, en defensa de las medidas adoptadas por Venezuela, buscando así, generar una esfera pública de apoyo.

Gráfico 3. Principales noticias publicadas

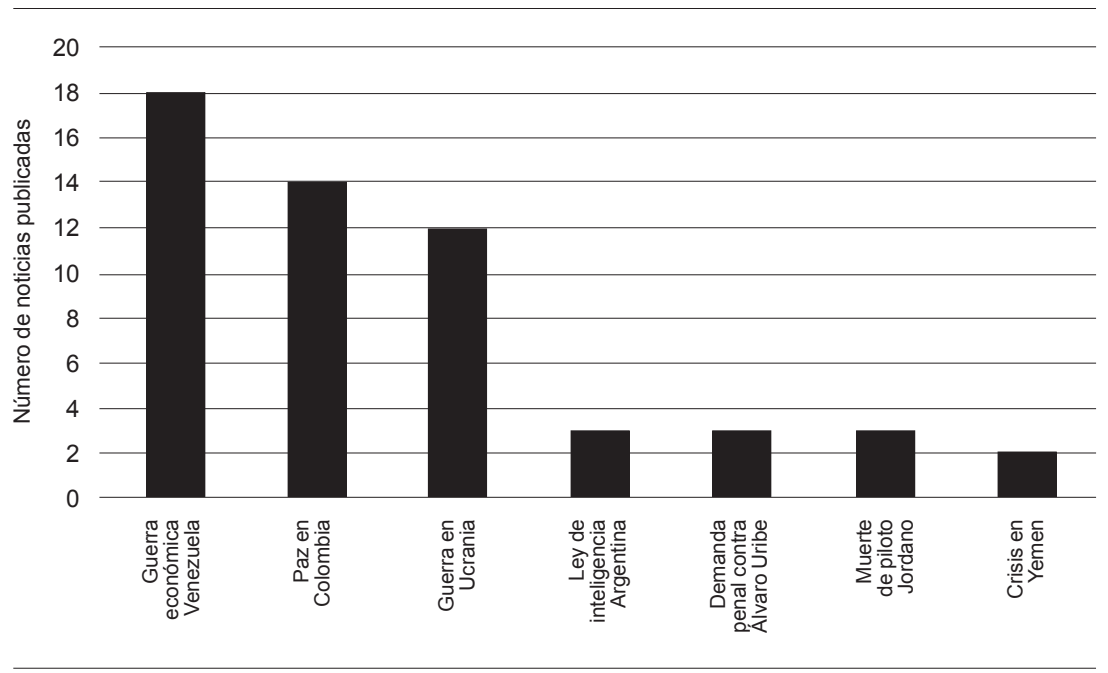

Fuente: elaboración propia.

Sin embargo, la investigación sobre la imagen de los países de la región conducida por la Fundación Imagen de Chile/IPSOS demuestra que Venezuela no ha logrado, incluso utilizando los medios de comunicación, una imagen positiva frente a la opinión publica extranjera ante a la pregunta: ¿Cuál es la opinión general que usted tiene de...? De esta manera, la opción muy favorable + favorable obtuvo un promedio de $22 \%$ entre los países de la América Latina, mientras que la tasa de muy desfavorable + desfavorable ha alcanzado valores de 65\% (Colombia) y 43\% (Perú), con un promedio de 39\% de rechazo.

Joseph Nye señala que para los Estados que han buscado promover su softpower a través de emisoras internacionales, como en el caso venezolano, es necesario la adopción de un conjunto de estrategias comunicacionales internacionales, mediante el cual, sectores sub-nacionales de la sociedad civil puedan participar directamente y destaca que el uso estas redes internacionales de comunicación para promover una 
Gráfico 4. Imagen de Venezuela en América Latina

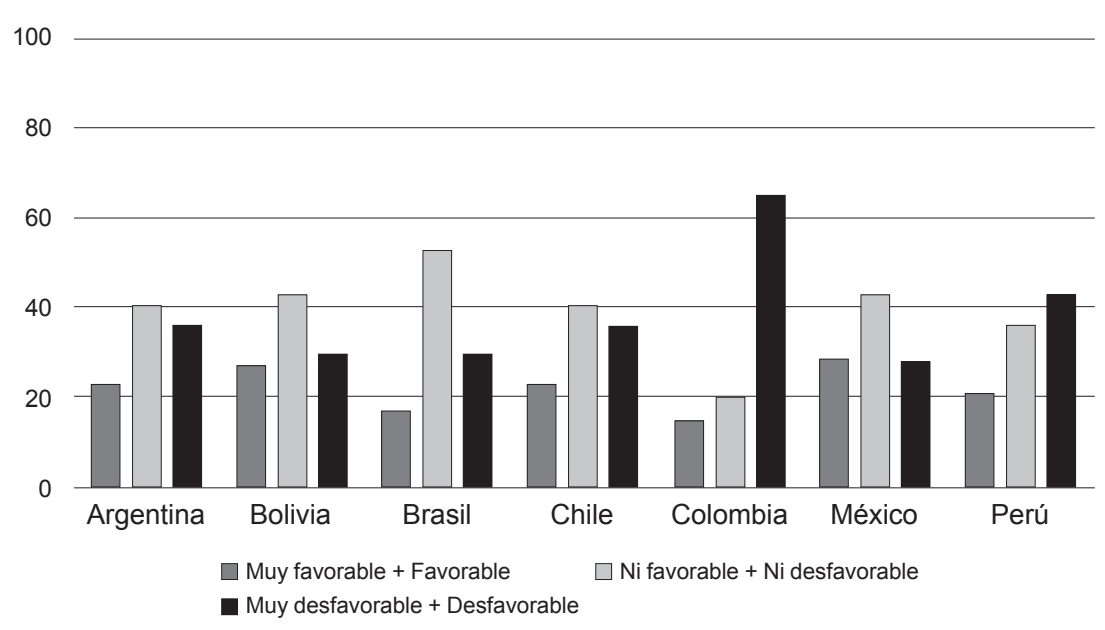

Fuente: Fundación Imagen de Chile / IPSOS (2013).

Diplomacia Pública es válido, sin embargo, si no existe una participación de demás actores sub-nacionales en la elaboración del contenido informativo donde la cultura, los valores y la políticas de un país tiene capacidad reducida de atracción debido el fuerte apelo propagandista estimulado por el gobierno nacional, como en el caso estudiado, el uso de una diplomacia pública para producir softpower a través de las redes internacionales de comunicación puede ser contraproducente. Y se puede producir todo lo contrario al deseado (Nye, 2008, p. 95).

Nye busca señalar que las noticias emitidas por estos instrumentos de comunicación internacional sean creíbles y de confianza internacional, debido que la información que aparenta ser propaganda suele ser despreciado, así como, puede llegar a ser contraproducente y auxiliar en la destrucción de la reputación internacional de un país. Como ejemplo, el autor señala el caso de Estados unidos donde las continuas afirmaciones entre cual Iraq tendría armas de destrucción en masa y estrecho vínculo con Al Qaeda, pueden haber ayudado a movilizar apoyo interno, pero la posterior noticia de estas noticias fueran exageración asestó un duro golpe para la credibilidad estadounidense (Nye, 2008, p. 101). 


\subsection{Venezuela y la Cyber Diplomacy}

Los Estados han buscado diversificar su fuerte gama de actuación, en especial, con la adquisición de nuevas tecnologías de comunicación internacional. El avance de internet en el mundo contemporáneo ha ocasionado cambios en la posturas de los Estados que, según señala Phalavi (2003), tienen un nuevo mecanismo para generar confianza y empatía, a través de la constitución de lo que el autor denomina atmosphere of trust en la red mundial de computadoras.

Phalavi señala que las nuevas tecnologías de comunicación se convierten en un instrumento cada vez más importante para la seguridad nacional, así como en un elemento que constituye parte del poder político, militar y económico (Phalavi, 2003). Además de esto, el avance tecnológico ha propiciado países de porte medio, como Irán y Canadá en el uso de nuevos mecanismos, a través del internet para promover la imagen del país y generar empatía internacional.

Según Phalavi, el objetivo de establecer un ambiente de confianza es el primer dispositivo que un Estado tiene en defensa de su seguridad nacional (Phalavi, 2003), por lo tanto, en el actual escenario de un mundo cada vez más interconectado e interdependiente, ser capaz de proyectar una imagen positiva a través de la red mundial de computadores, la llamada cyber-diplomacy, desarrolla un papel de crucial importancia para los Estados.

Josef Bátora en su estudio Public Diplomacy between home and abroad: Norway and Canada (2006), señala cómo el uso del internet por el ministerio de asuntos extranjeros de Canadá ha auxiliado la proyección y puntos de contacto entre la sociedad canadiense y el mundo, a través del desarrollo de un sitio web que promoviera discusiones sobre los más variables temas internacionales (Bátora, 2006). Esto, ha favorecido la imagen de Canadá internacionalmente. Por lo tanto, cuando los actores sociales son involucrados en la discusión de temas de política exterior resulta en ser más producente de que el uso exclusivo de propaganda internacional a través de los medios de comunicación para generar apoyo internacional 
En América Latina el uso de la cyber-diplomacy ha sido limitado en efectos de producir material que favorezca la imagen internacional del país, en el mejor de los casos, el objetivo es responder de manera reactiva las "amenazas" presentes en la red y no establecer un medio de contacto con aquello en lo que prevalezcan las discusiones sobre estos temas de modo que se genere una acción proactiva de promoción del softpower del país.

Así, en relación con el caso venezolano, podemos dar como ejemplo el mes de febrero de 2010, cuando el entonces presidente Hugo Chávez acusó la red social Twitter y otros medios de comunicación de estar asociados con el terrorismo y de promover la desestabilización de su gobierno; por lo tanto él había decidido unirse a esta red social abrir su propia cuenta para emprender una "batalla online" (Shaer, M., 2010).

El gráfico abajo señala la cantidad de seguidores de la cuenta del presidente Chávez, (@Chavezcandanga) en la red social Twitter, que abierta en abril de 2010, pasa en pocos meses, a tener más de un millón de seguidores transformándose en la cuenta más seguida en Venezuela, y mismo en el periodo posterior a la muerte de Chávez, su cuenta que no ha registrado más actividad, el número de seguidores ha aumentado aproximadamente en 200 mil usuarios.

\section{Gráfico 5. Hugo Chávez en Twitter}

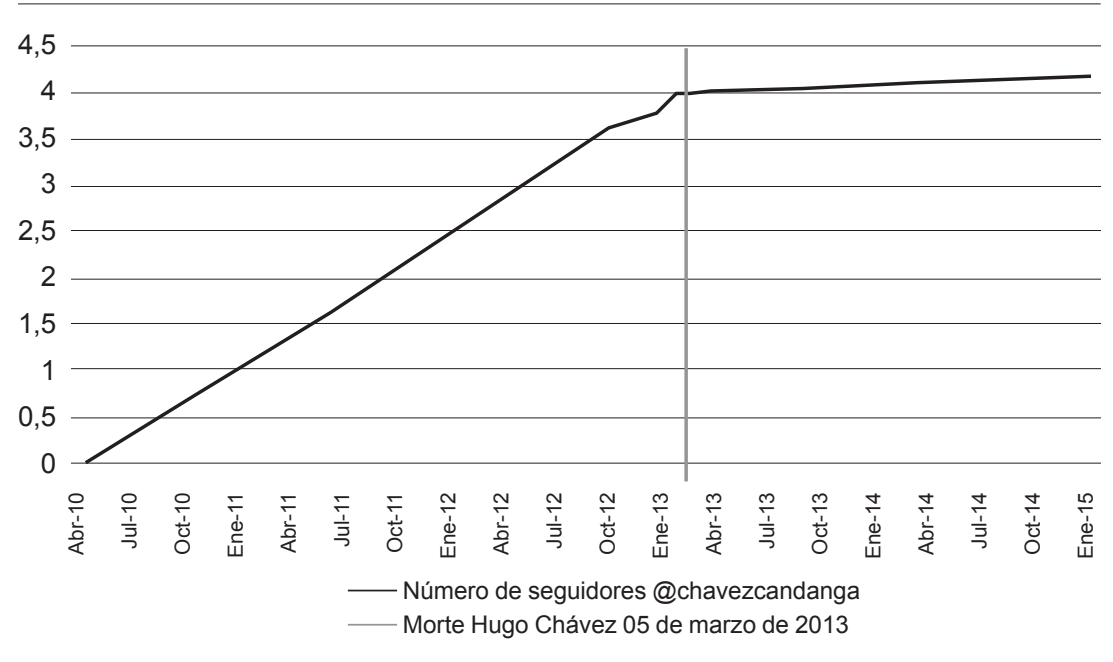

Fuente: Elaboración Propia a partir de la base de datos de tweetreach. 
A partir de 2010, el gobierno venezolano pasa a adoptar como estrategia comunicacional una fuerte presencia en las esferas virtuales. Actualmente, el gobierno venezolano mantiene una cuenta oficial institucional a cada uno de los 27 Ministerios de Gobierno, además de que diversos miembros de la administración pública, como ministros, militares y alcaldes poseen cuentas personales y utilizan la red social.

El fuerte culto a Chávez es un elemento presente en las cuentas institucionales del Gobierno Venezolano, fotos y menciones son aún recurrentes tanto en el perfil como en las mensajes oficiales emitidos por este medio de comunicación.

Así, como señala Craig Hayden (2013) en Venezuela es necesario entender que las nuevas Tecnología de la información y de las comunicaciones (TCI) han sido ampliamente utilizadas internamente y externamente por el gobierno venezolano como medios de inhibir a nuevos intentos de golpe de Estados, como el promovido en 2002, cual los medios de comunicación tradicionales, como la radio y la televisión, tuvieron una presencia importante en los hechos. En un país en el cual las audiencias son profundamente susceptibles a los medios de comunicación, es necesario incluirlo en el debate público sobre la diplomacia pública, sus variables el uso de las TICs y su influencia sobre la esfera política tanto nacional como internacional (Hayden, 2013).

El debate sobre la diplomacia pública se ha centralizado sobre los países desarrollados; sin embargo, aquello que denominamos cyberdiplomacy es una nueva metodología que busca generar apoyo y confianza internacional, en lo cual las TIC se ofrecen a los Estados en vías de desarrollo un crucial elemento en el cambio estratégico de cómo comunicarse internacionalmente.

No obstante, la muerte de Hugo Chávez, quien fue su principal figura y líder, ha representado una gran pérdida para la imagen internacional del país y del gobierno venezolano. Los profesores Valente \& Santoro (2006) señalan que el expresidente instigaba a construir una imagen internacional, que buscaba efectuar dos funciones a través 
de los medios de comunicación: por una parte, proyectar el liderazgo de Chávez sobre las Izquierdas Latinoamericana y los movimientos sociales, y, por otra parte, el poder de Venezuela sobre el continente.

Actualmente, el Presidente Nicolás Maduro, sin el carisma del expresidente, ha utilizado las redes sociales y los medios de comunicación con el objetivo de lograr respaldo político tanto nacional como internacional. Ejemplo de esto es el ingreso de una cuenta en la red social Twitter durante la campaña presidencial de 2013. Antes el entonces ministro del Poder Popular para Relaciones Exteriores (MRE); según el profesor Manuel Hidalgo, su candidatura ha sido impuesta por Chávez en reconocimiento a su lealtad, pero también al hecho de que Maduro no pertenecía a ninguno de los diferentes grupos rivales dentro del chavismo, además de su trabajo como Ministro, sus inclinaciones hacia la izquierda, y sus estrechas relaciones con los hermanos Castro en Cuba (Hidalgo, 2013, p. 319). Sin embargo, Maduro era una figura casi desconocida de las masas venezolanas, por este motivo, es destacable el uso de los medios de comunicación, especialmente los espacios virtuales como Twitter, en la tentativa de promover el liderazgo de Maduro en una Venezuela dividida y en un chavismo sin Chávez.

\section{Conclusiones}

La diplomacia pública representa para los Estados en vías de desarrollo, como Venezuela, la posibilidad de que la opinión pública internacional pueda conocer su postura frente a temas que le interesan. Lo que ayuda a reforzar sus posiciones logrando apoyo a sus demandas y capital político internacional. Mientras tanto, la diplomacia pública en dichos países encuentra limitaciones en condiciones estructurales de actuación, como es el caso de Telesur que no ha logrado un gran nivel de penetración en la opinión pública de América Latina. Por esto, urge un cambio en la estrategia de comunicación internacional por parte del gobierno de Nicolás Maduro utilizando mejor la estructura internacional de comunicación y profundizando el uso de la cyber-diplomacy, debido a la gran importancia que los nuevos medios de comunicación, especialmente, el internet ha ganado más espacio 
en detrimento de los medios tradicionales de comunicación como televisión.

Existen claras señales de que Telesur sirve como una herramienta de defensa del Estado venezolano frente a un "bloqueo" mediático sobre el país, pero el bajo nivel de aceptación de la imagen de Venezuela en el exterior demuestra que el gobierno venezolano post-Chávez no ha logrado comunicarse de manera efectiva con América Latina, el rasgo de propaganda pesa sobre el canal, por lo tanto, su principal medio de comunicación internacional ha fallado, hasta el momento, en lo que representa desafíos en materia de comunicación.

Por lo tanto, el principal desafío para Venezuela está en institucionalizar y ajustar estas estrategias de promover una diplomacia pública, ya que la falta de un plan institucional dificulta la implementación de un plan eficaz de comunicación internacional. Al largo de los 10 años de Telesur, los diversos cambios en el proyecto han dificultado enormemente la consolidación del proyecto. Aunque en el Estado venezolano ha demostrado intenciones en expandir Telesur y reajustar a este proyecto, a modo de alcanzar a nuevos públicos internacionales, pero que aún no ha logrado hacerlo.

\section{Referencias}

Aron, R. (2003). Peace and war: a theory of international relations. New Jersey: Transaction Publishers.

Burity, C. (2013). Mídia e Relações Internacionais: Diplomacia midiática no governo Lula (2003-2010). Recuperado de: http://www.sebreei. eventos.dype.com.br/resources/anais/21/136527 4115_ARQUIVO_CarolineBurity-GTPoliticaexterna.pdf

Bátora, J. (2006). Public Diplomacy between home and abroad: Norway and Canada, The Hague Journal of Diplomacy, 1, 53-80.

Cañizález, A. \& Lugo, J. (2007). Telesur: Estrategia geopolítica con fines integracionistas, Confines de relaciones internacionales y ciencia politica, 4(6), 58-64.

Carr, E. H. (2001). Vinte Anos de Crise: 1919-1939. Uma Introdução ao Estudo das Relações Internacionais. Brasilia: Universidade de Brasília, Instituto 
de Pesquisa de Relações Internacionais, Imprensa Oficial do Estado de São Paulo.

Hayden, C. (2013). Engaging Technologies: A comparative Study of U.S and Venezuelan Strategies of influence and Public Diplomacy, International Journal of Communication, 7, 1-25.

Gilboa, E., (2000). Media Coverage of International Negotiation: A Taxonomy of Levels and Effects, International Negotiation, 5(3), 543-568.

Gilboa, E. (2001). Diplomacy in the media age: Three models of uses and effects, Diplomacy \& Statecraft, 12(2), 1-28.

Gilboa, E. (2005). Media-Broker Diplomacy: When Journalists Become Mediators Critical Studies, Media Communication, 22(2), 99-120.

Hachten, W., \& Scotten, J. (2002). The World News Prism. Global Media in an Era of Terrorism (sexta edición). Wiley-Blackwell.

Delaney, R. (1968). Introducción. En Las comunicaciones en la diplomacia Moderna, (pp. 13-17), Ciudad de México: DIMELISA.

Lievingston, S. (1997). Clarifying the CNN effect: An Examination of Media Effects According to Type of Military Intervention. Harvard Collage. Recuperado de: http:/ /www.genocide-watch.org/images/1997Clarifying theCNNEffect-Livingston.pdf.

Hidalgo, M. (2013). The 2012 and 2013 Presidential elections in Venezuela. Electoral Studies, 34, 291-379.

Martínez Pandiani, G. (2006). Diplomacia Pública y Medios de Comunicación. Del Estado-Nacion a las cadenas mundiales de noticias, Buenos Aires: Ugerman Editor.

Nieto, A., \& Peña, M. (2008). La diplomacia pública: los medios informativos como instrumento de política exterior. México: Estudios Políticos.

Nye, J., Jr. (2008). Public Diplomacy and Soft Power. Annals of the American Academy of Political and Social Science, 616(1), 94-104.

Nye, J., Jr., \& Owens, W., A. (1996). American's Information Edge, Foreign Affairs, 75(2), 20-36.

Porcelli, E. (2013). Lo essencial es invisible a los ojos. El constructivismo en las relaciones internacionales. En E. Llenderroza (Coord.), Relaciones Internacionales Teorías y Debates, (pp. 65-105), Buenos Aires: EUDEBA.

Pahlavi, P. (2003). Cyber-Diplomacy: A New Strategy of Influence, En Law and Politics, Policy Analysis, Canadian Political Science Association General Meeting. 
Rai, M., \& Cottle. S. (2007). Global mediations; on the changing ecology of satellite television news. Global Media and Communication, 3(1), 51-78.

Snow, N. (2009). Rethink Public Diplomacy. En P. M. Taylor, Routledge handbook of Public Diplomacy. Nueva York: Routledge.

Shaer, M. (2010). Hugo Chavez. Will Reportedly Take the Revolution to Twitter. Christian Science Monitor. Recuperado de: http://www.csmonitor.com/ Innovation/Horizons/2010/0427/Hugo-Chavez-will-reportedlytake-the-revolution-to-Twitter

Valente, L., \& Santoro, M. (2014). A Diplomacia Midiática do Governo Hugo Chávez: Recuperado de: http://www.espacoacademico.com. br/060/60valentesantoro.htm 